\title{
On the characterization of asymptotic cases of the diffusion equation with rough coefficients and applications to preconditioning
}

\author{
Burak Aksoylu \\ Louisiana State University \\ Department of Mathematics \& \\ Center for Computation and Technology \\ Baton Rouge, LA 70803, USA \\ Horst R. Beyer \\ Louisiana State University \\ Center for Computation and Technology \\ Baton Rouge, LA 70803, USA
}

November 15, 2018

\begin{abstract}
We consider the diffusion equation in the setting of operator theory. In particular, we study the characterization of the limit of the diffusion operator for diffusivities approaching zero on a subdomain $\Omega_{1}$ of the domain of integration of $\Omega$. We generalize Lions' results to covering the case of diffusivities which are piecewise $C^{1}$ up to the boundary of $\Omega_{1}$ and $\Omega_{2}$, where $\Omega_{2}:=\Omega \backslash \bar{\Omega}_{1}$ instead of piecewise constant coefficients. In addition, we extend both Lions' and our previous results by providing the strong convergence of $\left(A_{\bar{p}_{\nu}}^{-1}\right)_{\nu \in \mathbb{N} *}$, for a monotonically decreasing sequence of diffusivities $\left(\bar{p}_{\nu}\right)_{\nu \in \mathbb{N}^{*}}$.
\end{abstract}

Mathematics Subject Classification (2000) 35J25, 47F05, 65J10, 65N99.

Keywords: Diffusion equation, diffusion operator, rough coefficients, singular diffusivities 


\section{Introduction}

The diffusion equation

$$
\frac{\partial u}{\partial t}=\operatorname{div}(p \operatorname{grad} u)+f
$$

describes general diffusion processes, including the propagation of heat, and flows through porous media. Here $u$ is the density of the diffusing material, $p$ is the diffusivity of the material, and the function $f$ describes the distribution of 'sources' and 'sinks'. The usage of $\bar{p}:=1 / p$ provides a convenient framework to study asymptotic cases where diffusivity approaches zero on an open subset of non-zero measure. Therefore, our definitions will be based on $\bar{p}$. This paper focuses on stationary solutions of (1.0.1) satisfying

$$
-\operatorname{div}((1 / \bar{p}) \operatorname{grad} u)=f .
$$

For instance, the fictitious domain method and composite materials are sources of rough coefficients; see the references in [6]. Important current applications deal with composite materials whose components have nearly constant diffusivity, but vary by several orders of magnitude. In composite material applications, it is quite common to idealize the diffusivity by a piecewise constant function and also to consider limits where the values of that function approach zero or infinity in parts of the material.

For the treatment of these questions, we use methods from operator theory. For this, we use a common approach to give (1.0.1) a well-defined meaning that, in a first step, represents the diffusion operator

$$
-\operatorname{div}(1 / \bar{p}) \operatorname{grad}
$$

as a densely-defined positive self-adjoint linear operator $A_{\bar{p}}$ in $L_{\mathbb{C}}^{2}(\Omega)$. As a result, (1.0.2) is represented by the equation

$$
A_{\bar{p}} u=f,
$$

where $f$ is an element of the Hilbert space, and $u$ is from the domain, $D\left(A_{\bar{p}}\right)$, of $A_{\bar{p}}$.

In our previous paper [1], we treat diffusivities from the class $\mathcal{L}$ consisting of 
$p \in L^{\infty}(\Omega)$ that are defined almost everywhere $\geqslant \varepsilon$ on $\Omega$ for some $\varepsilon>0$, where $\Omega \subset \mathbb{R}^{n}, n \in \mathbb{N}^{*}$, is some non-empty open subset. By use of Dirichlet boundary conditions, $\bar{p} \in \mathcal{L}$ induces a densely-defined, linear, self-adjoint, strictly positive operator $A_{\bar{p}}$ in $L_{\mathbb{C}}^{2}(\Omega)$. By assuming a weak notion of convergence in $\mathcal{L}$, we showed that the maps $\mathcal{S}$ and $\mathcal{T}$ defined by

$$
\begin{aligned}
\mathcal{S}(\bar{p}) & :=A_{\bar{p}}^{-1}, \\
\mathcal{T}(\bar{p}) & :=-(1 / \bar{p}) \nabla A_{\bar{p}}^{-1},
\end{aligned}
$$

for every $\bar{p} \in \mathcal{L}$ are strongly sequentially continuous.

For the case $n=1$ and bounded open intervals of $\mathbb{R}$, we were able to show stronger results that include also the asymptotic cases, except that where the asymptotic 'diffusivity' is almost everywhere infinite on the whole interval. We showed that $\mathcal{S}$ and $\mathcal{T}$ have unique extensions to sequentially continuous maps $\hat{\mathcal{S}}$ and $\hat{\mathcal{T}}$ in the operator norm on the set of a.e. positive elements of $L^{\infty}(\Omega) \backslash\{0\}$. In addition, an explicit estimate of the convergence behaviour of the maps is given,

$$
\hat{\mathcal{S}}(\bar{p})=\hat{\mathcal{S}}\left(\bar{p}_{\infty}\right)+\mathcal{O}\left(\left\|\bar{p}-\bar{p}_{\infty}\right\|_{1}\right) .
$$

Furthermore, we explicitly calculated $\hat{\mathcal{S}}$ and $\hat{\mathcal{T}}$. The knowledge of $\hat{\mathcal{S}}$ and $\hat{\mathcal{T}}$ for asymptotic $p$ is essential for the purpose of preconditioning. Since $\hat{\mathcal{S}}$ maintains continuity on $\partial \mathcal{L}$, the boundary value can be used as the dominant factor in a perturbation expansion for $\mathcal{S}(\bar{p})$ for $\bar{p} \in \mathcal{L}$. By rewriting (1.0.7), we arrive at an expression for a preconditioned operator:

$$
\begin{aligned}
& A_{\bar{p}}^{-1}=A_{\bar{p}_{\infty}}^{-1}+\mathcal{O}\left(\left\|\bar{p}-\bar{p}_{\infty}\right\|_{1}\right), \\
& A_{\bar{p}_{\infty}}^{-1} A_{\bar{p}}=I+\mathcal{O}\left(\left\|\bar{p}-\bar{p}_{\infty}\right\|_{1}\right) .
\end{aligned}
$$

For preconditioning purposes, in this paper, we study the boundary behaviour of $\mathcal{S}$ for $n>1$. We prove the strong convergence of $\mathcal{S}\left(\bar{p}_{\nu}\right)$ for any monotonically decreasing $\left(\bar{p}_{\nu}\right)_{\nu \in \mathbb{N}}$. We also characterize the associated limits for particular cases. The establishment of these results is the goal of the present article. As expected, the limits are structurally simpler. Therefore, utilizing the limits as preconditioners should lead to computationally feasible preconditioning. One such approach was taken by the first author in [2]. For showing effectiveness of the proposed preconditioner, one utilizes spectral equivalences. For the derivation of such equivalences, operator theory provides the natural framework. These questions are the subject for further study. 

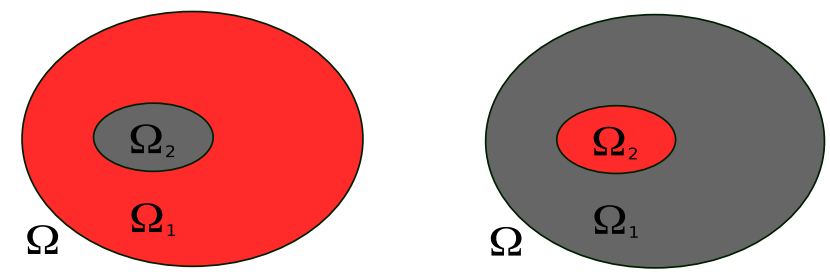

Fig. 1: Red and gray color indicate high and low diffusivity values, respectively. (Left) Lions' subdomain configuration. (Right) The configuration with diffusivity values swapped.

\section{Previous results and our improvements}

The treatment of the diffusion equation with piecewise constant discontinuous coefficient has been pioneered by J. L. Lions [7]. In his lecture notes, he considers the limit of the solution of (1.0.2) where the limit is associated to a one-parameter family of piecewise constant diffusivities $\left(\bar{p}_{\varepsilon}\right)_{\varepsilon \in(0, \infty)}$ approaching zero on a subdomain $\Omega_{1}$ of an open subset $\Omega$ of $\mathbb{R}^{n}, n \geqslant 1$. In this, the boundary of $\Omega_{1}$ intersects that of $\Omega$; see the left of Figure 1 .

Using a first order formulation of the diffusion operator, a similar piecewise constant one-parametric approach was used in [4, 5], but with diffusivities approaching infinity on a subdomain; see the right of Figure 1. By a simple scaling argument, it can be seen that the results based on such subdomain configuration can be reproduced from the Lions' configuration and vice versa.

In addition to our aforementioned one-dimensional results, in the previous paper [1], by assuming a weak notion of convergence in $\mathcal{L}$ for $n \geqslant 2$, we showed that the solution maps $\mathcal{S}$ and $\mathcal{T}$, defined in (1.0.5) and (1.0.6) respectively, are strongly sequentially continuous.

The basis of Lions' results provides an abstract lemma which derives a Laurent expansion for $\xi_{\varepsilon}$ in terms of $\varepsilon$ satisfying the equation

$$
s_{1}\left(\xi, \xi_{\varepsilon}\right)+\varepsilon s_{2}\left(\xi, \xi_{\varepsilon}\right)=\langle\xi \mid \eta\rangle
$$

for every $\xi \in X$. Here, $\eta \in X$ is given and $s_{1}, s_{2}$ are prescribed sesquilinear forms on the abstract Hilbert space $X$ satisfying certain conditions. The weak formulation of (1.0.2) corresponding to $\bar{p}_{\varepsilon}$ leads to this class of problems. Lions 
sketched the proof, only. For the convenience of the reader, this lemma is given in the appendix along with a full proof; see Lemma 5.0.4 and Lemma 5.0.5. In addition, Lions sketched the application of this lemma to the diffusion equation with piecewise constant coefficients as an example. Here, we extend Lions' results in various directions. In particular, we consider strong solutions of the operator equation instead of Lions' weak solutions. Note that the establishment of these results is based solely on the foundation provided in our preceding paper [1].

i) We generalize Lions' example to a theorem covering the case of diffusivities which are piecewise $C^{1}$ up to the boundary of $\Omega_{1}$ and $\Omega_{2}$, where $\Omega_{1}:=$ $\Omega \backslash \bar{\Omega}_{2}$ instead of piecewise constant coefficients. Note that for this, as is also the case in Lions' result, the source function $f$ is required to be an element of $W_{0, \mathbb{C}}^{1}(\Omega)$ which incorporates a regularity condition and a homogeneous boundary condition.

ii) In addition, we extend both Lions' and our previous results by providing the strong convergence of

$$
\left(\mathcal{S}\left(\bar{p}_{\nu}\right)\right)_{\nu \in \mathbb{N}^{*}}=\left(A_{\bar{p}_{\nu}}^{-1}\right)_{\nu \in \mathbb{N}^{*}},
$$

for a monotonically decreasing sequence $\bar{p}_{1}, \bar{p}_{2}, \ldots$ in $\mathcal{L}$; see Theorem 3.1.1. The coefficients in Lions' case, i.e., one parametric piecewise constant coefficients, automatically lead to a particular case of a monotonically decreasing sequence of diffusivities. Differently from Lions' case, our construction does not require a particular configuration of subdomains. On the other hand, differently to Lions, our theorem does not give a characterization of the corresponding the strong limit. Also, Lions shows convergence in the stronger $W^{1}$-norm as opposed to convergence in the $L^{2}$-norm, here.

\section{Preliminaries}

Definition 3.0.1. (Weak solutions) Let $X$ be a non-trivial complex Hilbert space, $A: D(A) \rightarrow X$ be a densely-defined, linear, self-adjoint and strictly positive operator in $X$. For $\eta \in X$, we call $\xi \in D\left(A^{1 / 2}\right)$ a weak solution of the equation

$$
A \xi=\eta
$$

if

$$
\left\langle A^{1 / 2} \xi \mid A^{1 / 2} \xi^{\prime}\right\rangle=\left\langle\eta \mid \xi^{\prime}\right\rangle
$$


for every $\xi^{\prime} \in D\left(A^{1 / 2}\right)$.

Remark 3.0.2. We note that the 'strong' solution of the equation (3.0.9), $\xi:=$ $A^{-1} \eta$, is also a weak solution of that equation. In addition, by the bijectivity of $A^{1 / 2}$, it follows the uniqueness of a weak solution. Hence $\xi \in D\left(A^{1 / 2}\right)$ is a weak solution of the equation (3.0.9) if and only if it is a strong solution of (3.0.9), i.e., if and only if $\xi \in D(A)$ and $A \xi=\eta$.

We define the diffusion operator as operator in $L_{\mathbb{C}}^{2}(\Omega)$ and give basic properties. Diffusion operators corresponding to diffusivities from the following large subset $\mathcal{L}$ of $L^{\infty}(\Omega)$ will turn out to be densely-defined, linear, self-adjoint operators.

Definition 3.0.3. We define the subset $\mathcal{L}$ of $L^{\infty}(\Omega)$ to consist of those elements $\bar{p}$ for which there are real $C_{1}, C_{2}$ satisfying $C_{2} \geqslant C_{1}>0$ and such that $C_{1} \leqslant \bar{p} \leqslant$ $C_{2}$ a.e. on $\Omega$. Note that the last also implies that $1 / \bar{p} \in \mathcal{L}$ and in particular that $1 / C_{2} \leqslant 1 / \bar{p} \leqslant 1 / C_{1}$ a.e. on $\Omega$.

Definition 3.0.4. For $\bar{p} \in \mathcal{L}$, we define the linear operator $A: D(A) \rightarrow L_{\mathbb{C}}^{2}(\Omega)$ in $L_{\mathbb{C}}^{2}(\Omega)$ by

$$
D(A):=\left\{u \in W_{0, \mathbb{C}}^{1}(\Omega):(1 / \bar{p}) \nabla_{w} u \in D\left(\nabla_{0}^{*}\right)\right\}
$$

and

$$
A u:=\nabla_{0}^{*}(1 / \bar{p}) \nabla_{w} u
$$

for every $u \in D(A)$.

Theorem 3.0.5. Let $\bar{p} \in \mathcal{L}$. Then $A$ is a densely-defined, linear, self-adjoint operator in $L_{\mathbb{C}}^{2}(\Omega)$.

Proof. See [1, Theorem 4.0.9].

Theorem 3.0.6. Let $\Omega$ be in addition bounded with a boundary of class $C^{2}$ and $\bar{p} \in C^{1}(\bar{\Omega}, \mathbb{R})$. Then

$$
D(A)=W_{0, \mathbb{C}}^{1}(\Omega) \cap W_{\mathbb{C}}^{2}(\Omega) .
$$

Proof. The statement is a simple consequence of elliptic regularity.

The following statement will be used in the proof of Theorem 3.1.1. Note that the domain of the quadratic form $q_{\eta}$ is given by $D\left(A^{1 / 2}\right)$, which is generally larger than $D(A)$. The same result holds if the domain of $q_{\eta}$ is restricted to $D(A)$. However, $D(A)$ depends heavily on the diffusivity, whereas, according to [1, Lemma 5.0.19], $D\left(A^{1 / 2}\right)=W_{0, \mathbb{C}}^{1}(\Omega)$. 
Theorem 3.0.7. (Variational formulation) Let $X$ be a non-trivial complex Hilbert space, $A: D(A) \rightarrow X$ be a densely-defined, linear, self-adjoint and strictly positive operator in $X$. Further, let $\eta \in X$ and $q_{\eta}: D\left(A^{1 / 2}\right) \rightarrow \mathbb{R}$ be defined by

$$
q_{\eta}(\xi):=\left\langle A^{1 / 2} \xi \mid A^{1 / 2} \xi\right\rangle-\langle\eta \mid \xi\rangle-\langle\xi \mid \eta\rangle
$$

for every $\xi \in D\left(A^{1 / 2}\right)$. Then $q_{\eta}$ assumes a unique minimum, of value

$$
-\left\langle\eta \mid A^{-1} \eta\right\rangle
$$

at $\xi=A^{-1} \eta$.

\subsection{Variational formulation}

In the following, we show the strong convergence of $\left(A_{\bar{p}_{\nu}}^{-1}\right)_{\nu \in \mathbb{N} *}$ for a monotonically decreasing sequence $\bar{p}_{1}, \bar{p}_{2}, \ldots$ in $\mathcal{L}$.

Lemma 3.1.1. Let $\bar{p}_{1}, \bar{p}_{2}, \ldots$ be a monotonically decreasing, i.e., such that for every $\nu \in \mathbb{N}^{*}$ the inequality $\bar{p}_{\nu+1}(x) \leqslant \bar{p}_{\nu}(x)$ holds for almost all $x \in \Omega$, sequence in $\mathcal{L}$. In addition, let $A_{1}, A_{2}, \ldots$ be the associated sequence of self-adjoint linear operators. Then the sequence $A_{1}^{-1}, A_{2}^{-1}, \ldots$ is strongly convergent to a positive bounded self-adjoint linear operator on $L_{\mathbb{C}}^{2}(\Omega)$.

Proof. For this, let $\nu \in \mathbb{N}^{*}$. Further, let $f \in L_{\mathbb{C}}^{2}(\Omega)$ and $q_{\nu, f}: W_{0, \mathbb{C}}^{1}(\Omega) \rightarrow \mathbb{R}$ be defined by

$$
\begin{aligned}
q_{\nu, f}(u): & =\left\langle A_{\nu}^{1 / 2} u \mid A_{\nu}^{1 / 2} u\right\rangle_{2}-\langle f \mid u\rangle_{2}-\langle u \mid f\rangle_{2} \\
& =\left\langle\nabla_{w} u \mid\left(1 / \bar{p}_{\nu}\right) \nabla_{w} u\right\rangle_{2, n}-\langle f \mid u\rangle_{2}-\langle u \mid f\rangle_{2}
\end{aligned}
$$

for every $u \in W_{0, \mathbb{C}}^{1}(\Omega)$.

$$
\left\|A_{\nu}^{1 / 2} f\right\|_{2}^{2}=\left\langle\nabla_{w} f \mid\left(1 / \bar{p}_{\nu}\right) \nabla_{w} f\right\rangle_{2, n}
$$

for every $f \in W_{0, \mathbb{C}}^{1}(\Omega)$. According to Theorem 3.0.7, $q_{\nu, f}$ assumes a unique minimum, of value

$$
-\left\langle f \mid A_{\nu}^{-1} f\right\rangle_{2}
$$

at $u_{\nu}:=A_{\nu}^{-1} f$. As a consequence, since $\bar{p}_{1}, \bar{p}_{2}, \ldots$ is monotonically decreasing, it follows that

$$
q_{\nu+1, f}(u) \geqslant q_{\nu, f}(u)
$$


for every $u \in W_{0, \mathbb{C}}^{1}(\Omega)$ and hence that

$$
q_{\nu+1, f}\left(u_{\nu+1}\right) \geqslant q_{\nu, f}\left(u_{\nu+1}\right) \geqslant q_{\nu, f}\left(u_{\nu}\right) .
$$

Hence it follows that

$$
\left\langle f \mid A_{\nu+1}^{-1} f\right\rangle_{2} \leqslant\left\langle f \mid A_{\nu}^{-1} f\right\rangle_{2}
$$

From the last, it follows that $A_{1}^{-1}, A_{2}^{-1}, \ldots$ is a monotonically decreasing sequence of positive bounded self-adjoint operators on $L_{\mathbb{C}}^{2}(\Omega)$ and as such strongly convergent to a positive bounded self-adjoint operator on $L_{\mathbb{C}}^{2}(\Omega)$.

\subsection{Generalization of Lions' Lemma}

We provide the structures satisfying the assumptions of Lemma 5.0.5 for treating the diffusion equation (1.0.4).

Theorem 3.2.1. Let $\Omega$ be a non-void bounded open subset of $\mathbb{R}^{n}$ with boundary of class $C^{2}, \Omega_{2} \subset \mathbb{R}^{n}$ be such that $\bar{\Omega}_{2} \subset \Omega$ and with boundary of class $C^{2}$. We define the closed subspace $X_{1}$ of $W_{0, \mathbb{C}}^{1}(\Omega)$ by the range of the isometric imbedding $\iota$ of $W_{0, \mathbb{C}}^{1}\left(\Omega_{2}\right)$ into $W_{0, \mathbb{C}}^{1}(\Omega)$ given by $\iota(f):=\hat{f}$ for every $f \in W_{0, \mathbb{C}}^{1}\left(\Omega_{2}\right)$, where

$$
\hat{f}(x):= \begin{cases}f(x) & \text { if } x \in \Omega_{2} \\ 0 & \text { if } x \in \Omega \backslash \Omega_{2} .\end{cases}
$$

In addition, let $p_{1}$ and $p_{2}$ be a.e. positive elements of $L_{\mathbb{C}}^{\infty}(\Omega)$ that vanish almost everywhere on $\Omega_{2}$ and $\Omega_{1}$, respectively, satisfy $p_{1}\left|\Omega_{1} \in C^{1}\left(\bar{\Omega}_{1}, \mathbb{R}\right), p_{2}\right| \Omega_{2} \in$ $C^{1}\left(\bar{\Omega}_{2}, \mathbb{R}\right)$ and for which there are $\alpha_{1}, \alpha_{2}>0$ such that $p_{j} \geqslant \alpha_{j}$ almost everywhere on $\Omega_{j}, j \in\{1,2\}$. Finally, let $s_{j}:\left(W_{0, \mathbb{C}}^{1}(\Omega)\right)^{2} \rightarrow \mathbb{C}$ be defined by

$$
s_{j}(f, g):=\left\langle\nabla_{w} f \mid p_{j} \nabla_{w} g\right\rangle_{2, n}
$$

for all $f, g \in W_{0, \mathbb{C}}^{1}(\Omega)$ and $j \in\{1,2\}$. Then $W_{0, \mathbb{C}}^{1}(\Omega), X_{1}, s_{1}, s_{2}$ satisfy the General Assumption 5.0.3.

Proof. By

$$
\|f\|:=\left(\sum_{k=1}^{n}\left\|\partial^{k} f\right\|_{2}^{2}\right)^{1 / 2}
$$


for all $f \in W_{0, \mathbb{C}}^{1}(\Omega)$, there is defined a norm \|\| on $W_{0, \mathbb{C}}^{1}(\Omega)$ that is equivalent to $\|\mid\|_{1}$. Therefore, without restriction, we can assume in the following that $W_{0, \mathbb{C}}^{1}(\Omega)$ is equipped with the norm \|\| . Further, by use of the inequalities

$$
\begin{aligned}
& \left|s_{j}(f, g)\right|=\left|\left\langle\nabla_{w} f \mid p_{j} \nabla_{w} g\right\rangle_{2, n}\right|=\left|\sum_{k=1}^{n}\left\langle\partial^{e_{k}} f \mid p_{j} \partial^{e_{k}} g\right\rangle_{2}\right| \\
& \leqslant\left\|p_{j}\right\|_{\infty} \sum_{k=1}^{n}\left\|\partial^{e_{k}} f\right\|_{2}\left\|\partial^{e_{k}} g\right\|_{2} \leqslant\left\|p_{j}\right\|_{\infty}\|f\|\|g\|
\end{aligned}
$$

for all $f, g \in W_{0, \mathbb{C}}^{1}(\Omega)$ and $j \in\{1,2\}$, it follows that $s_{1}$ and $s_{2}$ are bounded Hermitean positive sesquilinear forms. In addition, it follows that

$$
\begin{aligned}
& s_{1}(f, f)+s_{2}(f, f)=\left\langle\nabla_{w} f \mid\left(p_{1}+p_{2}\right) \nabla_{w} f\right\rangle_{2, n}=\sum_{k=1}^{n}\left\langle\partial^{e_{k}} f \mid\left(p_{1}+p_{2}\right) \partial^{e_{k}} f\right\rangle_{2} \\
& \geqslant \alpha \sum_{k=1}^{n}\left\|\partial^{e_{k}} f\right\|_{2}^{2}=\alpha\|f\|^{2}
\end{aligned}
$$

for every $f \in W_{0, \mathbb{C}}^{1}(\Omega)$, where $\alpha:=\min \left\{\alpha_{1}, \alpha_{2}\right\}$. Further, it follows for $f \in X_{1}$ that

$$
s_{2}(f, f)=\left\langle\nabla_{w} f \mid p_{2} \nabla_{w} f\right\rangle_{2, n} \geqslant C_{2}\left\langle\nabla_{w} f \mid \nabla_{w} f\right\rangle_{2, n}=C_{2}\|f\|^{2}
$$

and

$$
s_{1}(f, g)=\left\langle\nabla_{w} f \mid p_{1} \nabla_{w} g\right\rangle_{2, n}=0
$$

for every $g \in W_{0, \mathbb{C}}^{1}(\Omega)$. Further, we note that $s:=s_{1}+s_{2}$, as sum of two bounded Hermitean positive sesquilinear forms, is a bounded Hermitean positive sesquilinear form. In addition, as a consequence of (3.2.1), $s$ is positive definite and hence a scalar product for $W_{0, \mathbb{C}}^{1}(\Omega)$. Also

$$
\begin{aligned}
& s(f, f)=\left\langle\nabla_{w} f \mid\left(p_{1}+p_{2}\right) \nabla_{w} f\right\rangle_{2, n}=\sum_{k=1}^{n}\left\langle\partial^{e_{k}} f \mid\left(p_{1}+p_{2}\right) \partial^{e_{k}} f\right\rangle_{2} \\
& \leqslant \max \left\{\left\|p_{1}\right\|_{\infty},\left\|p_{2}\right\|_{\infty}\right\} \sum_{k=1}^{n}\left\|\partial^{e_{k}} f\right\|_{2}^{2}=\max \left\{\left\|p_{1}\right\|_{\infty},\left\|p_{2}\right\|_{\infty}\right\}\|f\|^{2}
\end{aligned}
$$


for every $f \in W_{0, \mathbb{C}}^{1}(\Omega)$. Hence it follows by (3.2.1) the equivalence of the norm that is induced on $W_{0, \mathbb{C}}^{1}(\Omega)$ by $s$ and \|\| . As a consequence, for $\omega \in L\left(W_{0, \mathbb{C}}^{1}(\Omega), \mathbb{C}\right)$, there is a unique $f \in W_{0, \mathbb{C}}^{1}(\Omega)$ such that

$$
\omega=s_{1}(f, \cdot)+s_{2}(f, \cdot) .
$$

In particular, if $\operatorname{ker} \omega \supset X_{1}$, this implies that

$$
\begin{gathered}
0=\omega(\hat{g})=s_{1}(f, \hat{g})+s_{2}(f, \hat{g})=s_{2}(f, \hat{g})=\left\langle\nabla_{w} f \mid p_{2} \nabla_{w} \hat{g}\right\rangle_{2, n} \\
=\left\langle f \mid \nabla_{0}{ }^{*} p_{2} \nabla_{w} \hat{g}\right\rangle_{2, n}=\left\langle\left(\left.f\right|_{\Omega_{2}}\right) \mid \nabla_{0, \Omega_{2}}{ }^{*}\left(\left.p_{2}\right|_{\Omega_{2}}\right) \nabla_{w, \Omega_{2}} g\right\rangle_{2, n, \Omega_{2}}
\end{gathered}
$$

for every $g \in W_{0, \mathbb{C}}^{1}\left(\Omega_{2}\right) \cap W_{\mathbb{C}}^{2}\left(\Omega_{2}\right)$, where an index $\Omega_{2}$ indicates the association of structures to $\Omega_{2}$, instead of $\Omega$. Since, according to Theorem 3.0.6,

$$
\left\{\nabla_{0, \Omega_{2}}{ }^{*}\left(\left.p_{2}\right|_{\Omega_{2}}\right) \nabla_{w, \Omega_{2}} g \in L_{\mathbb{C}}^{2}\left(\Omega_{2}\right): g \in W_{0, \mathbb{C}}^{1}\left(\Omega_{2}\right) \cap W_{\mathbb{C}}^{2}\left(\Omega_{2}\right)\right\}
$$

is dense in $L_{\mathbb{C}}^{2}\left(\Omega_{2}\right)$, the last implies that $f$ vanishes a.e. on $\Omega_{2}$ and hence that

$$
\omega=s_{1}(f, \cdot) .
$$

In addition, if $g \in W_{0, \mathbb{C}}^{1}(\Omega)$ is such that

$$
\omega=s_{1}(g, \cdot)
$$

it follows that

$$
\begin{aligned}
& 0=\left\langle\nabla_{w}(f-g) \mid p_{1} \nabla_{w} \hat{h}\right\rangle_{2, n}=\left\langle f-g \mid \nabla_{0} * p_{1} \nabla_{w} \hat{h}\right\rangle_{2, n} \\
& =\left\langle\left.(f-g)\right|_{\Omega_{1}} \mid \nabla_{0, \Omega_{1}}{ }^{*}\left(\left.p_{1}\right|_{\Omega_{1}}\right) \nabla_{w, \Omega_{1}} h\right\rangle_{2, n, \Omega_{1}}
\end{aligned}
$$

for every $h \in W_{0, \mathbb{C}}^{1}\left(\Omega_{1}\right) \cap W_{\mathbb{C}}^{2}\left(\Omega_{1}\right)$, where an index $\Omega_{1}$ indicates the association of structures to $\Omega_{1}$, instead of $\Omega$. Since, according to Theorem 3.0.6,

$$
\left\{\nabla_{0, \Omega_{1}}{ }^{*}\left(\left.p_{1}\right|_{\Omega_{1}}\right) \nabla_{w, \Omega_{1}} h \in L_{\mathbb{C}}^{2}\left(\Omega_{1}\right): h \in W_{0, \mathbb{C}}^{1}\left(\Omega_{1}\right) \cap W_{\mathbb{C}}^{2}\left(\Omega_{1}\right)\right\}
$$

is dense in $L_{\mathbb{C}}^{2}\left(\Omega_{1}\right)$, the last implies that $f-g$ vanishes a.e. on $\Omega_{1}$ and hence that $f-g \in X_{1}$.

We give a concrete example of the application of Lions' Lemma 5.0.4 to the diffusion equation (1.0.4). 
Corollary 3.2.2. Let $f \in W_{0, \mathbb{C}}^{1}(\Omega), \varepsilon>0, k \in \mathbb{N} \cup\{-1\}$, and $X_{1}$ as in Theorem 3.2.1. Restriction of a function to $\Omega_{i}$ is indicated by an addition of an index $i$.

(i) There is a unique $u_{\varepsilon} \in W_{0, \mathbb{C}}^{1}(\Omega)$ such that

$$
A u_{\varepsilon}=f .
$$

(ii) There is $C>0$ such that

$$
\left\|\sum_{j=-1}^{k} \varepsilon^{j} u_{j}-u_{\varepsilon}\right\|_{1} \leqslant C \varepsilon^{k+1},
$$

where $u_{-1} \in X_{1}$ and $u_{0}, \ldots, u_{k} \in W_{0, \mathbb{C}}^{1}(\Omega)$ are uniquely determined by

$$
\begin{aligned}
& -\nabla_{0}{ }^{*} p_{22} \nabla_{w} u_{-12}=f_{2}, \\
& -\nabla_{0}{ }^{*} p_{11} \nabla_{w} u_{01}=0,\left.\quad\left(\frac{\partial u_{01}}{\partial \nu}-\frac{\partial u_{-12}}{\partial \nu}\right)\right|_{\partial \Omega_{2}}=0, \quad u_{02}=0, \\
& -\nabla_{0}^{*} p_{11} \nabla_{w} u_{j 1}=0,\left.\quad\left(\frac{\partial u_{j 1}}{\partial \nu}-\frac{\partial u_{(j-1) 2}}{\partial \nu}\right)\right|_{\partial \Omega_{2}}=0 \\
& -\nabla_{0}^{*} p_{22} \nabla_{w} u_{j 2}=0,\left.\quad\left(u_{j 2}-u_{(j-1) 1}\right)\right|_{\partial \Omega_{2}}=0
\end{aligned}
$$

where $j \in\{1, \ldots, k\}$.

\section{Concluding remarks}

Based on the foundation provided by our previous paper [1], in this paper, we generalize Lions' results in various ways. Our results provide the existence of strong solutions of the operator equation instead of Lions' weak solutions. In particular, we generalize Lions' results to include diffusivities that are piecewise $C^{1}$ up to the boundary of $\Omega_{1}$ and $\Omega_{2}$, where $\Omega_{2}:=\Omega \backslash \bar{\Omega}_{1}$. Note that the geometric configuration is restricted to the case that the boundaries of $\Omega_{1}$ and $\Omega$ have a nonempty intersection. In the one dimensional case, a full characterization of the limiting inverse operator is given in our preceding paper [1], independent of the configuration. The other case corresponding to the right of Figure 1, i.e., when the boundary of $\Omega_{1}$ has an empty intersection with that of $\Omega$ with $n \geqslant 2$, is still largely 
open. On the other hand, for that configuration, a characterization of the limit of the discretized inverse operators with piecewise constant coefficients is given by the first author in [3] using linear finite element and finite volume methods.

\section{Appendix}

The following are the assumptions for Lions' abstract Lemma.

Assumption 5.0.3. Let $X$ be a non-trivial complex Hilbert space, $X_{1}$ a closed subspace of $X$ and $s_{1}: X^{2} \rightarrow \mathbb{C}, s_{2}: X^{2} \rightarrow \mathbb{C}$ be bounded sesquilinear forms on $X$, i.e., sesquilinear forms for which there are $C_{1}, C_{2} \geqslant 0$ such that

$$
\left|s_{i}(\xi, \eta)\right| \leqslant C_{i}\|\xi\|\|\eta\|
$$

for all $\xi, \eta \in X$ and $i \in\{1,2\}$. In addition, let $s_{1}, s_{2}$ be Hermitean, positive and satisfy the following conditions.

(i) There is $\alpha>0$ such that

$$
s_{1}(\xi, \xi)+s_{2}(\xi, \xi) \geqslant \alpha\|\xi\|^{2}
$$

for all $\xi \in X$,

(ii) 1) $s_{1}\left(\xi, \xi^{\prime}\right)=0$ for all $\xi \in X_{1}$ and $\xi^{\prime} \in X$,

2) for every $\omega \in L(X, \mathbb{C})$ such that $\operatorname{ker} \omega \supset X_{1}$, there is $\xi \in X$ such that $\omega=s_{1}(\xi, \cdot)$. In addition, if $\xi^{\prime} \in X$ is such that $\omega=s_{1}\left(\xi^{\prime}, \cdot\right)$, then $\xi^{\prime}-\xi \in X_{1}$.

(iii) There is $\alpha_{2}>0$ such that

$$
s_{2}(\xi, \xi) \geqslant \alpha_{2}\|\xi\|^{2}
$$

for all $\xi \in X_{1}$.

Lemma 5.0.4. Assume 5.0.3. Then, for every $\omega \in L(X, \mathbb{C})$ such that $\operatorname{ker} \omega \supset X_{1}$, there is a unique $\xi \in X$ such that $\omega=s_{1}(\xi, \cdot)$ and $s_{2}\left(\xi, \xi^{\prime}\right)=0$ for all $\xi^{\prime} \in X_{1}$. 
Proof. We note that, since $s_{2}$ is sesquilinear, Hermitean and positive, there is a uniquely determined positive self-adjoint $T_{2} \in L(X, X)$ such that

$$
s_{2}\left(\xi, \xi^{\prime}\right)=\left\langle\xi \mid T_{2} \xi^{\prime}\right\rangle
$$

for all $\xi, \xi^{\prime} \in X$. In the following, we denote by $P_{1}$ the projection onto $X_{1}$. Then the restriction $T_{21}$ of $P_{1} T_{2} P_{1}$ in domain and in image to $X_{1}$ is a positive selfadjoint element of $L\left(X_{1}, X_{1}\right)$. Further, since there is $\alpha_{2}>0$ such that for every $\xi \in X_{1}$

$$
s_{2}(\xi, \xi)=\left\langle P_{1} \xi \mid T_{2} P_{1} \xi\right\rangle=\left\langle\xi \mid T_{21} \xi\right\rangle \geqslant \alpha_{2}\|\xi\|^{2},
$$

$T_{21}$ is strictly positive and hence bijective. If $\omega$ is an element of $L(X, \mathbb{C})$ such that $\operatorname{ker} \omega \supset X_{1}$, then there is $\xi \in X$ such that $\omega=s_{1}(\xi, \cdot)$. Also for $\xi^{\prime \prime} \in X_{1}$, $\omega=s_{1}\left(\xi+\xi^{\prime \prime}, \cdot\right)$. Then

$$
s_{2}\left(\xi+\xi^{\prime \prime}, \xi^{\prime}\right)=0
$$

for all $\xi^{\prime} \in X_{1}$ if and only if

$$
\left\langle T_{21} \xi^{\prime \prime} \mid \xi^{\prime}\right\rangle=s_{2}\left(\xi^{\prime \prime}, \xi^{\prime}\right)=-s_{2}\left(\xi, \xi^{\prime}\right)=-\left\langle\xi \mid T_{2} \xi^{\prime}\right\rangle=-\left\langle P_{1} T_{2} \xi \mid \xi^{\prime}\right\rangle
$$

for all $\xi^{\prime} \in X_{1}$. Hence, (5.0.2) is satisfied for all $\xi^{\prime} \in X_{1}$ if

$$
\xi^{\prime \prime}=-T_{21}^{-1} P_{1} T_{2} \xi
$$

Further, if $\xi_{1}, \xi_{2} \in X$ are such that $\omega=s_{1}\left(\xi_{i}, \cdot\right)$ and $s_{2}\left(\xi_{i}, \xi^{\prime}\right)=0$ for all $\xi^{\prime} \in X_{1}$ and $i \in\{1,2\}$, then $\xi_{1}-\xi_{2} \in X_{1}$ and

$$
0=s_{2}\left(\xi_{1}-\xi_{2}, \xi_{1}-\xi_{2}\right) \geqslant \alpha_{2}\left\|\xi_{1}-\xi_{2}\right\|^{2} .
$$

Hence $\xi_{1}=\xi_{2}$.

Lemma 5.0.5. Assume 5.0.3. For $\eta \in X, \varepsilon>0$ and $k \in \mathbb{N} \cup\{-1\}$, it follows that

(i) There is a unique $\xi_{\varepsilon} \in X$ such that

$$
s_{1}\left(\xi, \xi_{\varepsilon}\right)+\varepsilon s_{2}\left(\xi, \xi_{\varepsilon}\right)=\langle\xi \mid \eta\rangle
$$

for all $\xi \in X$. 
(ii) There is $C>0$ such that

$$
\left\|\sum_{j=-1}^{k} \varepsilon^{j} \xi_{j}-\xi_{\varepsilon}\right\| \leqslant C \varepsilon^{k+1},
$$

where $\xi_{-1} \in X_{1}$ and $\xi_{0}, \ldots, \xi_{k} \in X$ are uniquely determined by $s_{2}\left(\xi, \xi_{-1}\right)=\langle\xi \mid \eta\rangle$ for all $\xi \in X_{1}$, $s_{1}\left(\xi, \xi_{0}\right)=\langle\xi \mid \eta\rangle-s_{2}\left(\xi, \xi_{-1}\right)$ for all $\xi \in X, s_{2}\left(\xi, \xi_{0}\right)=0$ for all $\xi \in X_{1}$, $s_{1}\left(\xi, \xi_{j}\right)=-s_{2}\left(\xi, \xi_{j-1}\right)$ for all $\xi \in X, s_{2}\left(\xi, \xi_{j}\right)=0$ for all $\xi \in X_{1}$, where $j \in\{1, \ldots, k\}$.

Proof. '(i)': Since $s_{1}, s_{2}$ are sesquilinear, Hermitean and positive, there are uniquely determined positive self-adjoint $T_{1}, T_{2} \in L(X, X)$ such that

$$
s_{i}\left(\xi, \xi^{\prime}\right)=\left\langle\xi \mid T_{i} \xi^{\prime}\right\rangle
$$

for all $\xi, \xi^{\prime} \in X$ and $i \in\{1,2\}$. Hence

$$
s_{1}\left(\xi, \xi^{\prime}\right)+\varepsilon s_{2}\left(\xi, \xi^{\prime}\right)=\left\langle\xi \mid\left(T_{1}+\varepsilon T_{2}\right) \xi^{\prime}\right\rangle
$$

for all $\xi, \xi^{\prime} \in X$. In addition, there is $\alpha>0$ such that

$$
s_{1}(\xi, \xi)+\varepsilon s_{2}(\xi, \xi)=\left\langle\xi \mid\left(T_{1}+\varepsilon T_{2}\right) \xi\right\rangle \geqslant \alpha\|\xi\|^{2}
$$

for all $\xi \in X$. As a consequence, $T_{1}+\varepsilon T_{2}$ is strictly positive and hence bijective. Therefore

$$
\xi_{\varepsilon}:=\left(T_{1}+\varepsilon T_{2}\right)^{-1} \eta
$$

satisfies

$$
s_{1}\left(\xi, \xi_{\varepsilon}\right)+\varepsilon s_{2}\left(\xi, \xi_{\varepsilon}\right)=\langle\xi \mid \eta\rangle
$$

for all $\xi \in X$. Further, if $\xi^{\prime} \in X$ is such that

$$
s_{1}\left(\xi, \xi^{\prime}\right)+\varepsilon s_{2}\left(\xi, \xi^{\prime}\right)=\langle\xi \mid \eta\rangle
$$

for all $\xi \in X$, then

$$
0=s_{1}\left(\xi^{\prime}-\xi_{\varepsilon}, \xi^{\prime}-\xi_{\varepsilon}\right)+\varepsilon s_{2}\left(\xi^{\prime}-\xi_{\varepsilon}, \xi^{\prime}-\xi_{\varepsilon}\right) \geqslant \alpha\left\|\xi^{\prime}-\xi_{\varepsilon}\right\|^{2}
$$


and hence $\xi^{\prime}=\xi_{\varepsilon}$.

'(ii)': For this, let $\xi_{-1}, \ldots, \xi_{k} \in X$ and

$$
\xi_{\varepsilon k}:=\sum_{j=-1}^{k} \varepsilon^{j} \xi_{j} .
$$

Then

$$
\begin{aligned}
& s_{1}\left(\xi, \xi_{\varepsilon k}\right)+\varepsilon s_{2}\left(\xi, \xi_{\varepsilon k}\right)=\sum_{j=-1}^{k} \varepsilon^{j} s_{1}\left(\xi, \xi_{j}\right)+\sum_{j=-1}^{k} \varepsilon^{j+1} s_{2}\left(\xi, \xi_{j}\right) \\
& =\varepsilon^{-1} s_{1}\left(\xi, \xi_{-1}\right)+\varepsilon^{k+1} s_{2}\left(\xi, \xi_{k}\right)+\sum_{j=0}^{k} \varepsilon^{j}\left[s_{1}\left(\xi, \xi_{j}\right)+s_{2}\left(\xi, \xi_{j-1}\right)\right]
\end{aligned}
$$

for every $\xi \in X$. Therefore, if

$$
s_{1}\left(\xi, \xi_{-1}\right)=0, s_{1}\left(\xi, \xi_{0}\right)=\langle\xi \mid \eta\rangle-s_{2}\left(\xi, \xi_{-1}\right), s_{1}\left(\xi, \xi_{j}\right)=-s_{2}\left(\xi, \xi_{j-1}\right),
$$

for all $\xi \in X$, where $j \in\{1, \ldots, k\}$, then

$$
s_{1}\left(\xi, \xi_{\varepsilon k}\right)+\varepsilon s_{2}\left(\xi, \xi_{\varepsilon k}\right)=\langle\xi \mid \eta\rangle+\varepsilon^{k+1} s_{2}\left(\xi, \xi_{k}\right)
$$

for all $\xi \in X$ and hence

$$
s_{1}\left(\xi, \xi_{\varepsilon k}-\xi_{\varepsilon}\right)+\varepsilon s_{2}\left(\xi, \xi_{\varepsilon k}-\xi_{\varepsilon}\right)=\varepsilon^{k+1} s_{2}\left(\xi, \xi_{k}\right)
$$

for all $\xi \in X$. In particular, this implies that

$$
\begin{aligned}
& \alpha\left\|\xi_{\varepsilon k}-\xi_{\varepsilon}\right\|^{2} \leqslant s_{1}\left(\xi_{\varepsilon k}-\xi_{\varepsilon}, \xi_{\varepsilon k}-\xi_{\varepsilon}\right)+\varepsilon s_{2}\left(\xi_{\varepsilon k}-\xi_{\varepsilon}, \xi_{\varepsilon k}-\xi_{\varepsilon}\right) \\
& =\varepsilon^{k+1} s_{2}\left(\xi_{\varepsilon k}-\xi_{\varepsilon}, \xi_{k}\right) \leqslant C_{2} \varepsilon^{k+1}\left\|\xi_{k}\right\|\left\|\xi_{\varepsilon k}-\xi_{\varepsilon}\right\|
\end{aligned}
$$

where $C_{2} \geqslant 0$ is such that

$$
\left|s_{2}(\xi, \eta)\right| \leqslant C_{2}\|\xi\|\|\eta\|
$$

for all $\xi, \eta \in X$. Hence it follows that

$$
\left\|\xi_{\varepsilon k}-\xi_{\varepsilon}\right\| \leqslant \frac{C_{2}}{\alpha}\left\|\xi_{k}\right\| \varepsilon^{k+1}
$$


In the following, we denote by $P_{1}$ the projection onto $X_{1}$. Then the restriction $T_{21}$ of $P_{1} T_{2} P_{1}$ in domain and in image to $X_{1}$ is a positive self-adjoint element of $L\left(X_{1}, X_{1}\right)$. Further, since there is $\alpha_{2}>0$ such that for every $\xi \in X_{1}$

$$
s_{2}(\xi, \xi)=\left\langle P_{1} \xi \mid T_{2} P_{1} \xi\right\rangle=\left\langle\xi \mid T_{21} \xi\right\rangle \geqslant \alpha_{2}\|\xi\|^{2},
$$

$T_{21}$ is strictly positive and hence bijective. Hence it follows for $\xi \in X_{1}$ and

$$
\xi_{-1}:=T_{21}^{-1} P_{1} \eta
$$

that

$$
\begin{aligned}
& s_{2}\left(\xi, \xi_{-1}\right)=\left\langle\xi \mid T_{2} T_{21}^{-1} P_{1} \eta\right\rangle=\left\langle P_{1} \xi \mid T_{2} T_{21}^{-1} P_{1} \eta\right\rangle=\left\langle\xi \mid P_{1} T_{2} P_{1} T_{21}^{-1} P_{1} \eta\right\rangle \\
& =\left\langle\xi \mid P_{1} \eta\right\rangle=\left\langle P_{1} \xi \mid \eta\right\rangle=\langle\xi \mid \eta\rangle .
\end{aligned}
$$

Further, if $\xi_{-1}^{\prime} \in X_{1}$ is such that

$$
s_{2}\left(\xi, \xi_{-1}^{\prime}\right)=\langle\xi \mid \eta\rangle
$$

for every $\xi \in X_{1}$, then

$$
0=s_{2}\left(\xi_{-1}^{\prime}-\xi_{-1}, \xi_{-1}^{\prime}-\xi_{-1}\right) \geqslant \alpha_{2}\left\|\xi_{-1}^{\prime}-\xi_{-1}\right\|^{2}
$$

and hence $\xi_{-1}^{\prime}=\xi_{-1}$. Further, since

$$
\langle\xi \mid \eta\rangle-s_{2}\left(\xi, \xi_{-1}\right)=0
$$

for every $\xi \in X_{1}$, it follows that

$$
\langle\eta \mid \cdot\rangle-s_{2}\left(\xi_{-1}, \cdot\right)
$$

is an element of $L(X, \mathbb{C})$ whose kernel contains $X_{1}$. Hence, by Lemma 5.0.4, there is a unique $\xi_{0} \in X$ such that

$$
s_{1}\left(\xi, \xi_{0}\right)=\langle\xi \mid \eta\rangle-s_{2}\left(\xi, \xi_{-1}\right)
$$

for every $\xi \in X$ and $s_{2}\left(\xi, \xi_{0}\right)=0$ for every $\xi \in X_{1}$. Finally, by Lemma 5.0.4, it follows recursively the existence and uniqueness of $\xi_{1}, \ldots, \xi_{k} \in X$ such that

$$
s_{1}\left(\xi, \xi_{j}\right)=-s_{2}\left(\xi, \xi_{j-1}\right) \text { for all } \xi \in X, s_{2}\left(\xi, \xi_{j}\right)=0 \text { for all } \xi \in X_{1},
$$

for $j \in\{1, \ldots, k\}$. 


\section{References}

[1] Aksoylu B and Beyer H. R. 2008, Results on the diffusion equation with rough coefficients, SIAM J. Math. Anal., submitted, also available at arXiv 0810.3427.

[2] Aksoylu B, Graham I G, Klie H, and Scheichl R 2008, Towards a rigorously justified algebraic preconditioner for high-contrast diffusion problems, Comput. Vis. Sci., 11, 319-331, doi:10.1007/s00791-008-0105-1 .

[3] Aksoylu B and Yeter Z, Conservative finite volume discretization of highcontrast diffusion equation and robust preconditioners, in preparation.

[4] Bakhvalov N S, Knyazev A V 1990, A new iterative algorithm for solving problems of the fictitious flow method for elliptic equations, Soviet Math. Dokl. 41, 481-485.

[5] Knyazev A V 1992, Iterative solution of PDE with strongly varying coefficients: Algebraic version, in: Beauwens R, De Groen P (eds) 1992, Iterative methods in linear algebra, Elsevier: New York, 85-89.

[6] Knyazev A and Widlund O 2003, Lavrentiev regularization + Ritz approximation = uniform finite element error estimates for differential equations with rough coefficients, Math. Comp., 72, 17-40.

[7] Lions J L 1973, Perturbations Singulieres Dans Les Problemes Aux Limites Et En Controle Optimal, Lecture Notes in Math., 323, Springer: Berlin. 